\title{
Intelligent Delivery of Multimedia Content in Mobile Environment
}

\author{
Aleksandar Karadimce ${ }^{1}$, Reza Malekian ${ }^{1,2, *}$ and Abdul Hanan Abdullah ${ }^{2}$ \\ ${ }^{1}$ Department of Computer Science and Engineering, University for Information Science and Technology "St. Paul the Apostle", Ohrid, \\ Republic of Macedonia \\ ${ }^{2}$ Faculty of Computer Science and Information Systems, Universiti Teknologi Malaysia, Johor, Malaysia
}

\begin{abstract}
Process of delivering multimedia learning objects such as text, video, images, audio, and animation requires real-time streaming of multimedia content from the M-learning systems to the mobile users. With enhancing the integration methods of the mobile devices into the learning systems, we have possibility to allow easy storing, recording and delivering of multimedia contents to the students in real-time. In this paper, we will present an intelligent mobile learning (M-learning) system for delivery of multimedia content depending of the context-aware conditions. This paper considers the significance of high QoS requirements for applications that are essential to achieve higher continuity of real-time multimedia contents. In this research, we have considered the best queuing packet delivery algorithm to provide appropriate multimedia contents to improve required bandwidth for user's communications.
\end{abstract}

Keywords: Multimedia Computing, M-learning, Quality of Service, User Profile.

\section{Introduction}

The existing perspective for mobile learning (M-learning) consists of presenting a lecture or e-books with lot of pages of text and graphics [1], delivered on a very small screen. However, with the introduction of new mobile information technology [2] and pervasive expansion of wireless technology, these conventional techniques will be replaced the brand-new mobile learning [3]. In recent years, mobile learning is a field which combines mobile computing and electronic learning (e-learning) [3], have provided more interactive and personalized instruction based on the learner's context [4] and learner cognitive profile [5]. Content adaptation [6] provides the most suitable applications according to students' computing context [7], referred to devices, network, location, and time, which affect students' mobile access of learning content [3]. In particular, network layer Quality of Service (QoS) parameters, that have huge influence on the bandwidth allocation in the process of multimedia content delivery [8] are lower start up delay and reduced end-to-end delay. M-learning systems deliver an interactive environment [9], through the right tools and support, research given in [10] and [11] show that students can retain significantly more and achieve a greater level of skill and performance. In order to reach higher endurance of multimedia, this creates new challenge in the area of content management and content delivery [12]. The process of continued delivery of multimedia content is highly dependent from efficient communication channels in M-learning systems in order to ease the transfer of multimedia content to the mobile users. In this paper we have researched the use of different queuing packet delivery algorithms that will provide interactive communication and personalized multimedia content for the mobile clients. This paper is organized as follows: Section II presents our proposed intelligent M-learning system architecture. Section III presents the simulation results of delivery of multimedia content in M-learning environment. Finally, Section IV concludes the paper.

\footnotetext{
*Corresponding author e-mail: reza.malekian@ieee.org
} 


\section{Intelligent M-Learning System Architecture}

The proposed architecture of intelligent M-learning system is presented in Figure 1. The scenario is consisted of two similar laboratories for M-learning that have wireless access point router and mobile clients. This way all the students using different mobile devices (smart phones, tablets and mobile phones [13]) can easy connect, through the wireless router, to the multimedia streaming server [14].

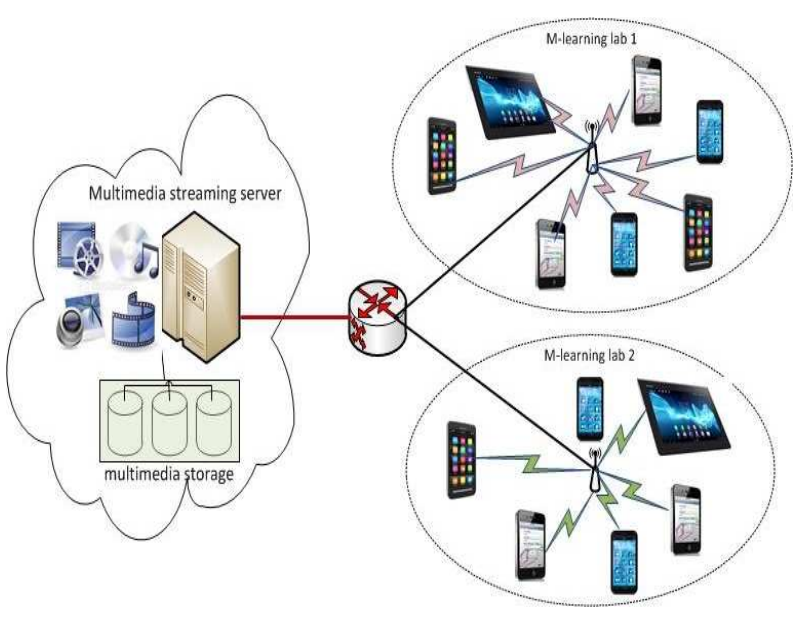

Fig. 1: System architecture of intelligent M-learning system

From the presented architecture we can determine that the bottleneck for multimedia delivery is from the multimedia streaming server to the switch. This happens because of the increased heavy load of multimedia data that needs to be delivered to the two M-learning laboratories in the same time. In order to estimate the bandwidth congestion in the presented architecture we have conducted experiments by modeling the proposed network and defining possible scenarios using software simulation tool. Therefore, the simulation is a brilliant tool for studying performance and identifying the Quality of Service (QoS) factors that have influence on multimedia contents delivery.

\section{Simulation Results for Delivery of Multimedia Content in M-Learning Environment}

The proposed system architecture of intelligent M-learning system is been developed in the OPNET IT Guru simulator, see Figure 2. Because it provides a comprehensive development environment with a full set of tools including model design, simulation, data collection, data analysis and support on the modeling of communication networks [15]. We have used the Discrete Event Simulation (DES) [16] because it enables modeling in a more accurate and realistic way. It creates an extremely detailed, packet-by-packet model for predicting the activities of the network. Multimedia streaming server (application configuration) is configured for streaming real-time audio, video and multimedia data [22](text or images), similar like in the real M-learning systems [18]. Existing research have discovered the Interaction is one of the most significant factors in assessing the quality of e-learning [9]. In order to simulate the M-learning laboratory we have configured two subnets that contain wireless access router and 9 to 5 mobile clients. The requests for multimedia content are streamed from the Multimedia streaming server to the mobile clients in the M-learning laboratory 1 or 2. Mobile clients using the profile configuration have been settled to three different cognitive learning skills: visualizer, verbalizer and bimodal users [19]. This way every mobile user depending of his cognitive learning style can receive appropriate multimedia content [20].

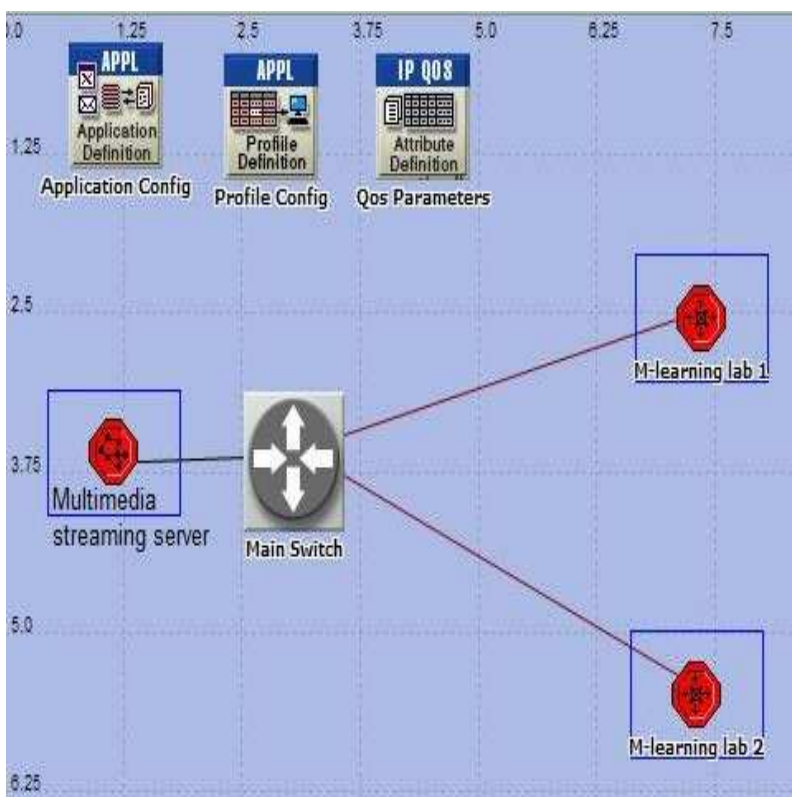

Fig. 2: Simulation of the intelligent M-learning system

From our preliminary simulations we have noticed that the bottleneck for multimedia delivery from multimedia streaming server is the communication link from the server to the switch. For that purpose we have established QoS parameters for the bottleneck link in order to improve the multimedia delivery. Depending of the used QoS queuing packet delivery algorithm we have formulated three different simulation scenarios. The first scenario is using First In First Out (FIFO) packet queuing 
algorithm [21] for the bottleneck link between the multimedia streaming server and the switch. The second scenario is using Priority Queuing (PQ) algorithm [22] to determine the delivery of multimedia packets. Finally, the third scenario is using the Weighted-Fair Queuing (WFQ) [23] algorithm for transferring of multimedia packets. The network simulator was configured to run one hour of multimedia content in the established M-learning system for the three different scenarios (FIFO, PQ and WFQ algorithms). The blue line represents the results from the FIFO packet queuing algorithm simulation, the red line represents the results from the PQ packet queuing algorithm simulation and the green line gives the results from WFQ packet queuing algorithm simulation. Analyzing the Wireless Local Area Network (WLAN) delay from the M-learning system in the three different scenarios, see Figure 3, we conclude that the FIFO packet queuing algorithm is generating the biggest delay. On the other side, the scenario with PQ packet queuing algorithm improves WLAN delay with $25 \%$ of total delay in comparison to the FIFO packet queuing algorithm. The lowest WLAN delay of $29 \%$ is accomplished with the scenario WFQ packet queuing algorithm in the M-learning system.

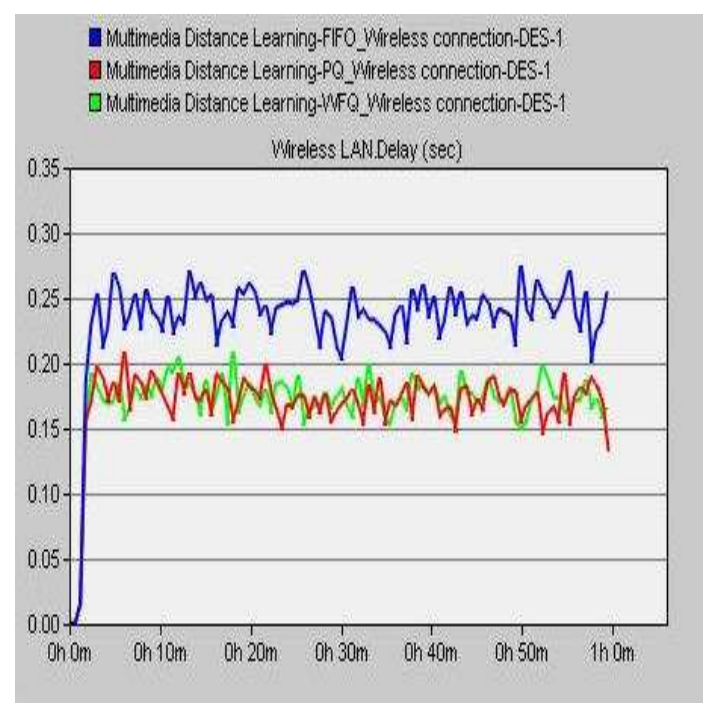

Fig. 3: Results from WLAN delay

The results from the Voice traffic QoS parameter, average packet delay, for the three different scenarios are shown in Figure 4. Considering the verbalizer cognitive perception, in the process of voice delivery we have discovered that the lowest average delay is in the scenario that uses WFQ packet queuing algorithm. By comparison of the WFQ scenario with the FIFO scenario, it enhances $37,5 \%$ of the voice average packet delay. Nonetheless, the PQ packet queuing algorithm has shown also an enhancement of $31.25 \%$ in voice average packet delay compared with FIFO scenario. The results from the Video

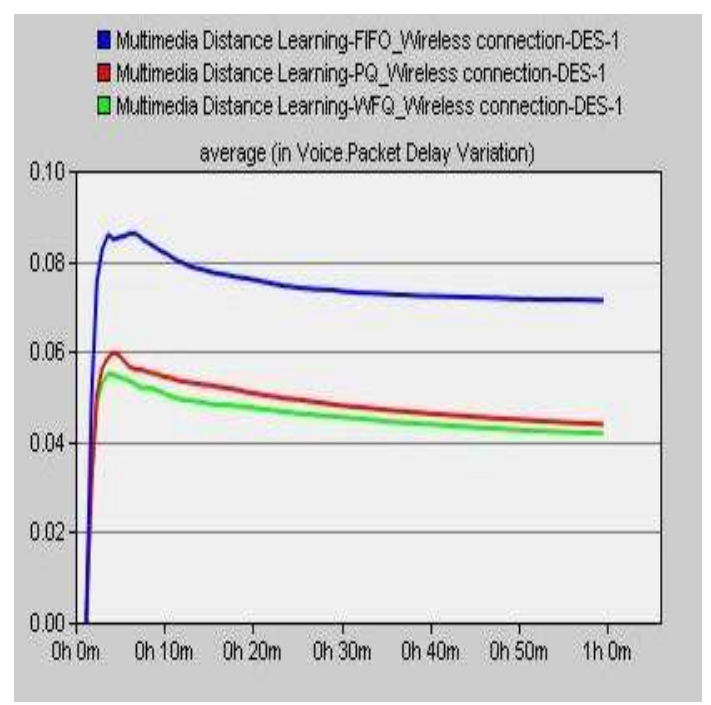

Fig. 4: Results from voice average packet delay

traffic QoS parameter, end-to-end average packet delay, for the three different scenarios are shown in Figure 5. The visualizer cognitive perception given by the video delivery has shown lowest average delay in the both of scenarios the PQ and the WFQ packet queuing algorithm.

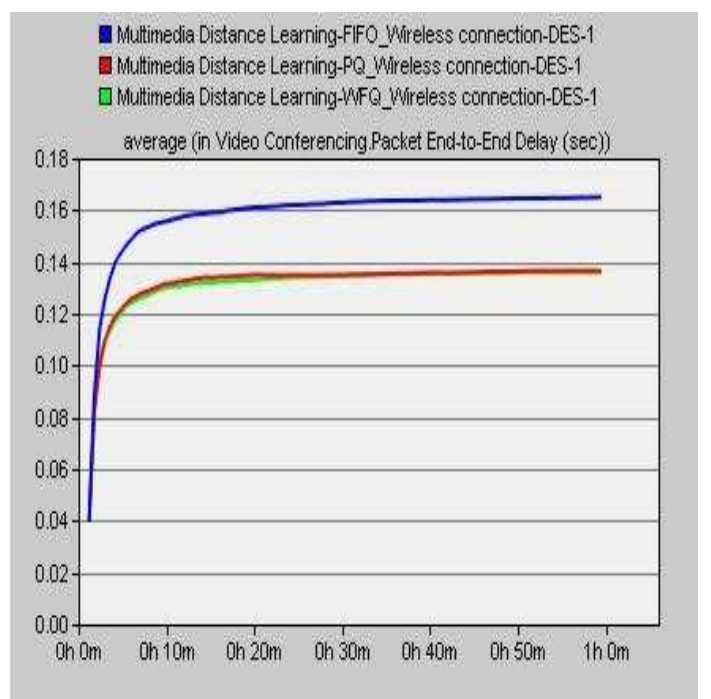

Fig. 5: Results from Video end-to-end average packet delay simulator

In conclusion we can see that almost the results are achieved for the both of scenarios the PQ and the WFQ packet queuing algorithm. While the FIFO algorithm for 
packet delivery that represents standard communication network has shown the poorest results for all of the monitored QoS parameters. Therefore, we can determine that the M-learning environment that is consisted with the existence of priority and weighted-fair queuing in the communication link have provided improved delivery of multimedia traffic.

\section{Conclusion and Future Work}

In conclusion the PQ and the WFQ packet queuing algorithm scenarios have shown decreased delay that has led to improved collaborative response to the mobile clients. Eventually, this means efficient and increased learner perception and satisfaction from the M-learning system that will provide better quality of learning. Priority packet queuing delivery algorithm utilizes scalable video/voice to provide a graceful degradation of the multimedia content in case of bandwidth shortages. The results from this research can be focused on further promotion of potential for m-learning using the cloud computing for training students in the universities [24], [25].

\section{Appendix A}

A review of distributed Queue algorithm analysis presented by (Kobliakov et al.) in [26] appears below. We use the results presented by (Kobliakov et al.) in [26] for simulation and evaluation of intelligent delivery of multimedia content in M-learning environment. Let suppose that M-learning system has infinite queue of packets intervals sets that have autonomous similar distributed random variables. The proposed system is modeled with stable queue that has the following condition assigned:

$$
\Lambda<\lambda
$$

where $\Lambda$ is the queue mean number of received packets in the interval through the M-learning system, in the duration $1 / \mu$. The transmission rate was calculated as maximal value of $\lambda$, the $\lambda$ max, by means of determining under which inequity (1) holds. Let the bottleneck interval is spitted into $S$ and $N$ slots during the operation of system. During the frame either one or none sets may arrive into the distributed queue, thus the arrival of one set is

$$
\Lambda=P_{r}\{\text { set }\}=1 e^{-\lambda}-\lambda e^{-\lambda}+q_{0} e^{-\lambda}+q_{1} e^{-\lambda}
$$

Let

$$
T(x)=\sum_{k=2}^{\infty} T_{k} \frac{x^{k}}{k !} e^{-x}+T_{0} e^{-x} q_{0}+T_{1} x e^{-x} q_{1}
$$

Where $T_{k}$ is the mean number of frames needed to serve collision set consisting of $($ kgeq0 $)$ requests. It can be shown, that $\psi=1 / \mu$ can be computed in the following way

$$
\psi=\frac{T(\lambda)}{1-e^{-\lambda}-\lambda e^{-\lambda}+q_{0} e^{-\lambda}+q_{1} \lambda e^{-\lambda}}
$$

From equations (1) - (4) we determine, that proposed queuing system is constant to deliver for such values of $\lambda$, as long as this inequity stands

$$
T(\lambda)<1
$$

Considering the equations (1) - (5), the transmission rate $R$ is calculated as $R(S, N)=\lambda_{\max }(S+N)$, where $\lambda_{\max }$ is the maximal $\lambda$ under which the following holds

$$
T\left(\frac{\lambda}{S}\right)<\frac{N}{2 S}
$$

\section{Acknowledgement}

This research is done under the Post-Doctoral Fellowship Scheme at Universiti Teknologi Malaysia for the Project "Mobility Management Based on Resource Reservation Over Mobile Application (ROMA) Approach over Mobile Internet Protocol Version 6 Networks". The authors are grateful to the anonymous referee for a careful checking of the details and for helpful comments that improved this paper.

\section{References}

[1] S. Joseph, and M. Uther, Mobile language learning with multimedia and multi-modal interfaces, in Proc. Proceeding of IEEE International Workshop on Wireless and Mobile Technologies in Education (ICHIT'06), 124-128 (2006).

[2] D. Gil, J. Andersson, M. Milrad, and H. Sollervall, Towards a Decentralized and Self-Adaptive System for M-Learning Applications, Proceeding of IEEE Seventh International Conference on Wireless, Mobile and Ubiquitous Technology in Education (WMUTE), 162-166 (2012).

[3] S. R. Yang, and Y. P. Lim, Towards Content Adaptation for Mobile Learning, Proceeding of 2nd International Conference on Education and Management Technology, IPEDR IACSIT Press, Singapore, 77-81 (2011).

[4] H. J. La, and S. D. Kim, A Conceptual Framework for Provisioning Context-aware Mobile Cloud Services, Proceeding of IEEE 3rd International Conference on Cloud Computing, 466 - 473 (2010).

[5] V. H. Muntean, and G. M. Muntean, A novel adaptive multimedia delivery algorithm for increasing user quality of experience during wireless and mobile e-learning, Proceeding of IEEE International Symposium on Broadband Multimedia Systems and Broadcasting (BMSB), 1-6 (2009). 
[6] O. Pettersson, and D. Gil, On the Issue of Reusability and Adaptability in M-learning Systems, Proceeding of 6th IEEE International Conference on Wireless, Mobile, and Ubiquitous Technologies in Education, 161-165 (2010).

[7] J. Wang, and J. L. Kourik, Delivering database knowledge with web-based labs, Proceeding of American Society of Business and Behavioral Sciences, ASBBS in Las Vegas. 923-931 (2012).

[8] K. R. Laghari, T. T. Pham, H. Nguyen, and N. Crespi, QoM: A new quality of experience framework for multimedia services, Proceeding of IEEE Symposium on Computers and Communications (ISCC), 851-856 (2012).

[9] I. Jung, The Dimensions of E-Learning Quality: From the Learner's Perspective, Published in Educational Technology Research and Development, 59, 445-464 (2011).

[10] R. S. Shariffudin, C. H. Julia-Guan, T. Dayang, N. Mislan, and M. F. Lee, Mobile Learning Environments for Diverse Learners in Higher Education, IJFCC, 2012, 32-35 (2012).

[11] M. Saranya, and M. Vijayalakshmi, Interactive Mobile Live Video Learning System in Cloud Environment, Proceeding of IEEE-International Conference on Recent Trends in Information Technology, ICRTIT, 673- 677 (2011).

[12] L. J. Zhang, EIC Editorial: Introduction to the Body of Knowledge Areas of Services Computing, IEEE Transactions on services computing, 1, 62-74 (2008).

[13] D. Gil de la Iglesia, Designing a Decentralized Distributed Self-Adaptive System in M-Learning Activities, Proceeding of 7th IEEE International Conference on Wireless, Mobile and Ubiquitous Technology in Education, 296-300 (2012).

[14] C. G. Cristian, V. A. Poenaru, and G. Suciu, Multimedia content distribution between core network routers using Peer-to-Peer (P2P), Proceeding of 19th Telecommunications Forum (TELFOR), 254-257 (2011).

[15] M. Hammoshi, and R. Al-Ani, Using OPNET to teach students computer networking subject, Tikrit Journal of Pure Science, 15, 281-292 (2010).

[16] J. Zhang, Discrete Event Simulation Enabled High Level Emulation of a Distribution Centre, Proceeding of 14th International Conference on Computer Modelling and Simulation (UKSim), 470-475 (2012).

[17] Reza Malekian, Abdul Hanan Abdullah, Traffic Engineering Based on Effective Envelope Algorithm on Novel Resource Reservation Method over Mobile Internet Protocol Version 6, International Journal of Innovative Computing, Information and Control, 8, 6445-6460 (2012)

[18] P. Lavin-Mera, P. Moreno-Ger, and B. Fernandez-Manjon, Development of Educational Videogames in m-Learning Contexts, Proceeding of Second IEEE International Conference on Digital Games and Intelligent Toys Based Education, 44-51 (2008).

[19] R. E. Mayer, and L. J. Massa, Three facets of visual and verbal learners: Cognitive ability, cognitive style and learning preference, Journal of Educational Psychology, 12, 833-846 (2003).

[20] V. Palo, M. Sinatra, P. Di Bitonto, and T. Roselli, How cognitive styles affect the e-learning process, Proceeding of 12th IEEE International Conference on Advanced Learning Technologies, 359-363 (2012).

[21] M. Karsten, FIFO Service with Differentiated Queueing, Proceeding of Seventh ACM/IEEE Symposium on Architectures for Networking and Communications Systems (ANCS), 122-133 (2011).
[22] Y. Qian, Z. Lu, and Q. Dou, QoS scheduling for NoCs: Strict Priority Queueing versus Weighted Round Robin, Proceeding of IEEE International Conference on Computer Design (ICCD), 52-59 (2010).

[23] M. F. Homg, W. T. Lee, K. R. Lee, and Y. H. Kuo, An adaptive approach to weighted fair queue with QoS enhanced on IP network, Proceeding of IEEE Region 10 International Conference on Electrical and Electronic Technology (TENCON), 1, 181-186 (2001).

[24] M. Wang, and J. W. P. Ng, Intelligent Mobile Cloud Education. Smart anytime-anywhere learning for the next generation campus environment, Proceeding of Eighth International Conference on Intelligent Environments, 149156 (2012).

[25] Q. Shuai, and Z. Ming-quan, Cloud computing promotes the progress of M-learning" , in Proc. of the IEEE conference of Uncertainty Reasoning and Knowledge Engineering (URKE), 162-164 (2011).

[26] A. Kobliakov, A. Turlikov, and A. Vinel, Distributed queue random multiple access algorithm for centralized data networks, Proceeding of the 10th IEEE International Symposium on Consumer Electronics, 290-295 (2006). 


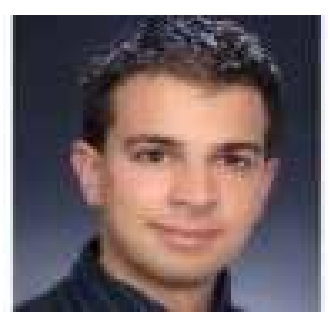

Aleksandar Karadimce

Aleksandar Karadimce

is currently working in

the University of Information

Science and Technology "St.

Paul the Apostle" in Ohrid

as a Research and Teaching

Assistant for Information Science and Technology. In the same time I'm $\mathrm{PhD}$ candidate at the Faculty of Computer Science and Engineering University Ss Cyril and Methodius in Skopje. Received his MSc degree in 2011 at University Ss. Cyril and Methodius, Faculty of Mechanical Engineering - Skopje. On the same university in 2007 he received his BSC degree on Faculty of Electrical Engineering and Information Technologies Skopje. Member of IEEE and ACM.

Reza Malekian

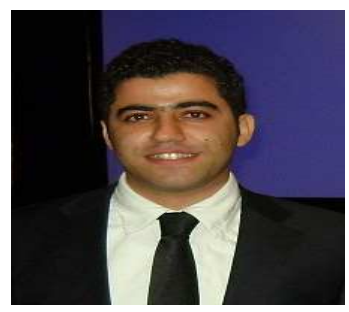

received Ph.D. in Computer

Science from Universiti Teknologi Malaysia (Erasmus partner) in 2012, M.Eng. (with honor) in Information Technology Engineering from Iran University of Science and Technology in 2008, and B.Eng in Computer Engineering from North University in 2006.

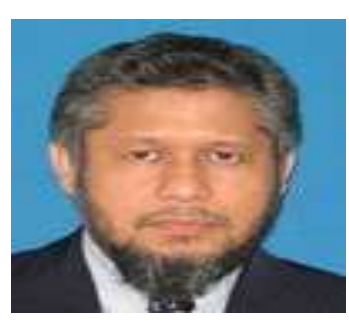

\section{Abdul}

Hanan Abdullah obtained his $\mathrm{PhD}$ degree from Aston University in Birmingham, United Kingdom in 1995. He has been the dean at the Faculty of Computer Science and Information systems for seven years from 2004 to 2011. Currently he is heading Pervasive Computing Research Group, a research group under K-Economy Research Alliances. His research interests include wireless sensor networks, mobile ad hoc networks, network security and next generation networks. 